\title{
Are there capacity limitations in symmetry perception?
}

\author{
LIQIANG HUANG and HAROLD PASHLER \\ University of California, San Diego, La Jolla, California \\ and \\ JUSTIN A. JUNGE \\ Yale University, New Haven, Connecticut
}

\begin{abstract}
Previous researchers have proposed that there are two types of symmetry detection: one based on crude preattentive symmetry judgments and another based on detailed scrutiny of individual parts (Barlow \& Reeves, 1979; S. E. Palmer \& Hemenway, 1978; Royer, 1981). Four experiments were conducted to examine capacity limits in different symmetry judgments. Observers were required to discriminate between random patterns and approximate symmetry (Experiments 1 and 3) or between perfect and approximate symmetry (Experiments 2 and 4). The patterns were divided into two sets of dots, presented either simultaneously or successively. A comparison of accuracy under these two presentation conditions suggested that symmetry detection involves an analysis that is spatially parallel but coarse, regardless of either task difficulty or task type (detecting symmetry vs. detecting asymmetry).
\end{abstract}

Mirror symmetry is often highly salient to human observers (Barlow \& Reeves, 1979), and the detection of symmetry may play an important role in mammalian vision in general. Some writers view symmetry as one of the most important aspects of early visual analysis (Wagemans, 1995). The present article examines questions at the interface between symmetry perception and visual attention that inquire into the existence and nature of capacity limitations in the detection of symmetry.

A number of writers have proposed a two-stage model of symmetry detection (S. E. Palmer \& Hemenway, 1978; Royer, 1981; for recent models, see Dakin \& Hess, 1997; Gurnsey, Herbert, \& Kenemy, 1998; Rainville \& Kingdom, 2000; van der Helm \& Leeuwenberg, 1996). According to these models, when observers are required to discriminate between a symmetrical pattern and a random pattern, a preattentive symmetry analysis takes place. For finer judgments (e.g., discriminating between random displays and random displays with partial symmetry, or discriminating between perfect symmetry and slightly perturbed symmetry), an attention-demanding pointwise matching process is undertaken. Various results have been interpreted to argue that symmetry detection is

This work was supported by National Institute of Mental Health Grant R01-MH45584. The authors are grateful to Cathleen Moore, Johan Wagemans, Chris Tyler, and one anonymous reviewer for useful comments; to an anonymous reviewer of a previous version of this manuscript for suggestions that helped us to develop the strategy employed in this paper; and to Kristen Sakamoto and Noriko Coburn for assistance with all aspects of the work. Correspondence concerning this article should be addressed to H. Pashler, Department of Psychology 0109, University of California, San Diego, La Jolla, CA 92093 (e-mail: hpashler@ucsd.edu). preattentive for crude and easy symmetry detection (Barlow \& Reeves, 1979; Locher \& Wagemans, 1993) but not for finer and more difficult judgments (Barlow \& Reeves, 1979; Foster, 1991; Royer, 1981; Wenderoth, 1997; for a review of the two-stage models, see Wagemans, 1995).

The concept of preattentive processing has several different aspects that can sometimes be dissociated from each other. Preattentive processing is often assumed to be fast, automatic, and unlimited in capacity, as opposed to slower, more flexible, limited-capacity controlled processing. The paradigmatic case of preattentive processing is usually assumed to be pop-out in visual search, where a target differs on a salient featural dimension from homogeneous distractors. Thus, the notion of preattentive processing actually involves three potentially separate questions (Hochstein \& Ahissar, 2002; Pashler, 1998).

Several different methods have been proposed and used to identify whether a process is preattentive. Julesz offered an operational definition of preattentive processing, according to which any property that can be detected in a very brief display is said to be detected preattentively (Julesz, 1981, p. 28). In the literature on symmetry detection, evidence has been presented to show that determination of crude symmetry can be performed very quickly (Barlow \& Reeves, 1979; Locher \& Wagemans, 1993; Royer, 1981; Wenderoth, 1997), whereas detecting more subtle symmetry or asymmetry requires longer exposure. These data appear consistent with the two-stage model (S. E. Palmer \& Hemenway, 1978; Royer, 1981).

Another question is whether symmetry perception is automatic and hence immune to the effects of mental set. If the observers are aware of some property of the stimuli, can they voluntarily adjust their symmetry percep- 
tion to exploit this property? Recent work has suggested that symmetry detection is sensitive to the cued (Pashler, 1990) and most frequent (Wenderoth, 1994) orientation of the axis of symmetry, suggesting that even crude symmetry judgments are not completely automatic in this sense. The third issue, and the focus of the present investigation, relates to capacity limitations: Will symmetry perception worsen when the amount of stimulus information to be processed at any given instant is increased? The evidence just described says little about whether processing is limited in capacity or not. One recent article has speculated whether symmetry perception is parallel across the whole visual field (van der Helm \& Leeuwenberg, 1996). However, to our knowledge this has never been demonstrated empirically. One approach that has been used to examine capacity limitations in many tasks is to determine the effect of display set size on response time (RT). This technique was popularized in the area of visual search by Treisman (Treisman \& Gelade, 1980). The only direct and explicit measurement of this effect we are familiar with involved a task in which multiple items each had its own symmetry axis (Olivers \& van der Helm, 1998). Thus, the question of whether the symmetry perception of one pattern has a limited capacity or not (Does the processing quality (or speed) decrease when the amount of available information increases?) is still an open issue.

There are several problems with the use of set-size measurements to assess processing-capacity limitations in symmetry detection. First, in symmetry detection there is no widely agreed "unit" of stimulus load, and some have doubted that a dot by dot comparison takes place (Barlow \& Reeves, 1979; Tapiovaara, 1990). Second, if detection is imperfect, set-size effects may arise simply from decision noise, as we will describe in more detail below (Eckstein, Thomas, J. Palmer, \& Shimozaki, 2000; J. Palmer, 1994; Shaw, 1984). Third, increases in RTs with increased display sizes may reflect eye movements (after all, eye movements are inherently serial). Fourth, relevant information is usually kept constant in search (only one target), whereas the quantity of irrelevant information (the number of distractors) varies. In the symmetry perception task, if we want to keep the difficulty (percentage of noisy dots) constant, both relevant and irrelevant information increase with the number of elements in the display. Thus, performance may be affected in both positive and negative directions.

For the reasons just described, the use of a method other than RT slope analysis would seem to be recommended. In the present study, we use a different method that also originates from visual search studies.

\section{Decision Noise and the Successive/Simultaneous Comparison Method}

As noted above, in a visual search or similar experiment, if all items are analyzed with the same fixed degree of accuracy or efficiency (i.e., if there are no capacity limitations), increasing the number of items will increase the overall probability of an error even in the absence of any capacity limitation (J. Palmer, 1994; Shaw, 1984). A strong test of capacity limitations in symmetry detection requires a different type of experiment.

A different and more powerful test of capacity limitations was first introduced by Eriksen and Spencer (1969) and Shiffrin and Gardner (1972). This test involves assessing the accuracy with which a judgment can be made when the entire display is presented simultaneously (SIM), as compared with the situation in which different parts of the same display (subdisplays) are exposed successively (SUCC). In each condition, any given subdisplay is presented for the same period of time; thus, for each part of the stimulus, the time available for processing is identical across the SIM and SUCC conditions. If detection is capacity-limited, there should be a substantial advantage in the SUCC display, because capacity can then be allocated to a subset of the display at any given time. If detection has an unlimited capacity and operates in parallel, on the other hand, there should be no difference in accuracy between SUCC and SIM displays. This method has been widely used in studies of visual search but not, to our knowledge, in research on symmetry perception. In the present study, a display of dots was presented either in its entirety (SIM) or divided equally into two parts that were exposed in succession (SUCC). The comparison between performance in the SUCC and SIM conditions allows one to ask about capacity allocation while holding display content - and thus statistical decision noise - constant (Duncan, 1980; Gardner \& Joseph, 1975). In the studies reported here, we used a very brief stimulus presentation $(100 \mathrm{msec})$ that did not allow time for eye movements, precluding a serial strategy induced by eye movements. Also, the fourth problem mentioned above (that both relevant and irrelevant information increase when the quantity of stimuli increase) does not undermine the rationale for the SUCC/SIM comparison, because both relevant and irrelevant information are equated in the two conditions.

As mentioned above, we cannot assume in advance what may constitute a privileged unit in symmetry perception. To avoid such assumptions in the present study, we apply two methods of dividing a display into two subdisplays to be presented sequentially in the SUCC condition. In Experiments 1 and 2, the random dot pattern display is divided into two spatial regions, each presented successively. This method halves the spatial extent of the display and keeps density within the occupied region constant. In Experiments 3 and 4, we randomly select half of the dot pairs to compose one frame and assign the other half to the second frame (thereby halving density while keeping spatial extent constant). Each mirror-symmetric pair of dots was always assigned to the same frame in the SUCC trial, so subjects did not have to compare information across frames.

In summary, the present study seeks to clarify capacity limitations in symmetry perception by systematically comparing two modes of presentation: simultaneous pre- 
sentation of the whole display for a given duration versus dividing the display into two parts and presenting each successively for the same duration. This comparison was performed for two different symmetry perception tasks. According to the two-stage model, as the task requires more precise spatial judgments, capacity limits are expected to emerge; thus, we predict that observers should do better in SUCC conditions in tasks requiring precision.

\section{METHOD}

\section{Observers}

Observers were volunteers from the University of California, San Diego, CA. All had normal or corrected-to-normal vision. There were 12 observers in each of Experiments 1-4, 48 observers in total.

\section{Apparatus}

Stimuli were presented on a 1,024 × 768 MAG DX-15T color monitor driven by a Pentium IV $1.8 \mathrm{G}$ personal computer. Responses were recorded from two adjacent keys using a standard keyboard. The observers viewed the displays from a distance of about $60 \mathrm{~cm}$.

\section{Stimuli}

Stimuli are shown in Figure 1. Each target display consisted of a region (not marked with any border on the screen) that measured $6.3 \mathrm{~cm}$ high $\times 6.3 \mathrm{~cm}$ wide $\left(6^{\circ} \times 6^{\circ}\right.$ visual angle, based on a typi- cal viewing distance of $60 \mathrm{~cm}$ ) with white dots embedded in the square. Each dot measured $0.08 \times 0.08 \mathrm{~cm}$. In symmetrical trials, the entire display was symmetrical about a vertical axis bisecting the screen (the axis was not shown). There were 200 dots in each display. The background was black (luminance $<0.2 \mathrm{~cd} / \mathrm{m}^{2}$ ), the dots were white (luminance $>30 \mathrm{~cd} / \mathrm{m}^{2}$ ), and the contrast between stimuli and background was high ( $>98 \%)$. In Experiments 1 and 3, the observers were required to discriminate between a random dot pattern and an approximately symmetric display (easy condition, $10 \%$ random dots; hard condition, 25\% random dots). In Experiments 2 and 4, the observers were required to discriminate between perfectly and approximately symmetric displays (easy condition, $25 \%$ random dots; hard condition, $10 \%$ random dots). Different proportions of approximate symmetric displays (hard condition and easy condition) were separated into different blocks and counterbalanced across observers.

In Experiments 1 and 2, for the SUCC trials, the whole display was divided into two halves (top half and bottom half, divided by a horizontal middle line), and each was presented as one frame. The vertical position of each half was randomized in the area of the SIM display, holding the density constant but halving the area in any given SUCC frame. In Experiments 3 and 4, in SUCC frames, half of the dot pairs were randomly assigned to the first frame and the other half assigned to the second frame. Each mirror-symmetric pair of dots was always assigned to the same frame in SUCC trial, so observers did not have to compare positions of dots in different frames. The proportion of dot pairs that were symmetric was the same in each frame of the SUCC displays and in SIM displays. Thus, area was held constant while density was halved in the SUCC frames.

\section{Experiments 1 and 3: Approximate Symmetry vs. Random Pattern}
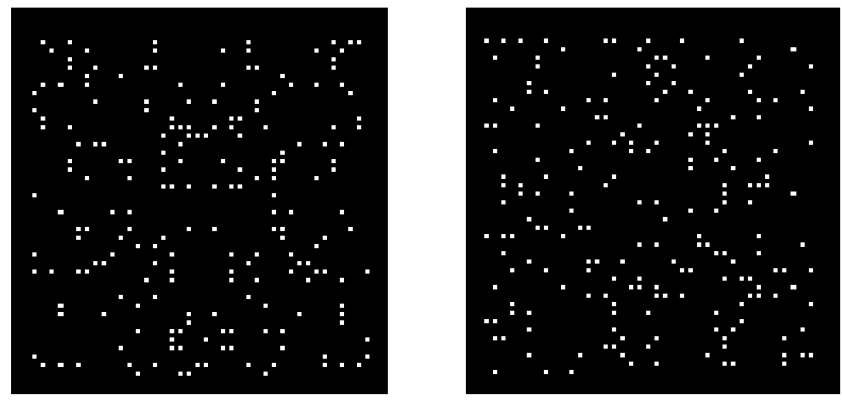

\section{Experiments 2 and 4: Perfect Symmetry vs. Approximate Symmetry}

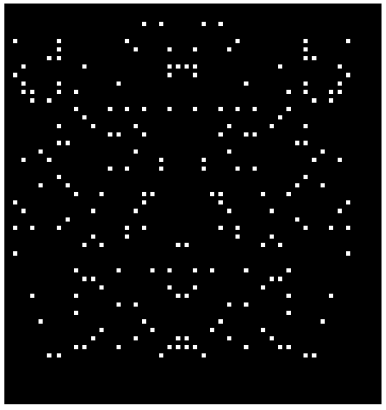

Symmetric

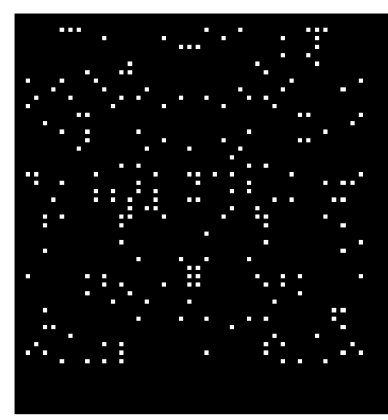

Asymmetric

Figure 1. Kinds of stimuli used in this study. In Experiments 1 and 3 (top figures), the observers discriminated between approximate symmetry and random patterns. In Experiments 2 and 4 (bottom figures), the observers discriminated between perfect and approximate symmetry. 
The mask consisted of a dense array of white dots filling the region occupied by the patterns being judged (but extending no farther).

\section{Procedure}

The procedure is shown in Figure 2. Observers were told that displays were symmetric on half of the trials. Each trial began with a small green fixation cross presented in the center of the screen. Observers were instructed to fixate the cross, which remained present for $400 \mathrm{msec}$. The cross was followed by a short blank interval $(400 \mathrm{msec})$, which was then followed by the display. In the SIM display, the entire display was presented for $100 \mathrm{msec}$ and then replaced by the mask $(100 \mathrm{msec})$. In the SUCC display, the two displays were presented successively. Each was individually and locally masked after $100 \mathrm{msec}$. The interstimulus interval between the first frame and the second frame in the SUCC display was $400 \mathrm{msec}$, and the duration of the mask was $100 \mathrm{msec}$. The SIM and SUCC displays were randomly mixed within each block.

In all the experiments, after the entire display was presented (both frames in the SUCC condition), observers responded by pressing one of two adjacent keys with fingers of the right hand. They were told to respond as accurately as possible. (Response keys were enabled only after stimulus presentation was concluded, and subjects were instructed to wait until all stimuli were shown.) A positive or negative sound was played to provide feedback on the accuracy of each response. Each observer performed 12 blocks of 60 trials, with the first 2 blocks excluded as practice.

\section{RESULTS}

We used signal detection measures $\left(d^{\prime}\right)$ instead of percent correct for our analysis to avoid individual variations in decision criteria. In the present design, each condition had separate hits and false alarms, so the computation of $d^{\prime}$ was straightforward. Figure 3 shows the average value of $d^{\prime}$ in each of the different experiments and conditions, and Figure 4 provides a summary of the $d^{\prime}$ results for Experiments $1-4$. Table 1 shows the difference in $d^{\prime}$ s between SIM and SUCC conditions for each of the experiments, along with the results of a one-way analysis of variance (ANOVA) testing the SUCC-SIM difference. In Experiments 1 and 2 (density constant; area halved), there was no significant difference between SIM and SUCC presentations, regardless of whether the task was to detect approximate symmetry (Experiment 1 ) or a small asymmetry (Experiment 2) and regardless of difficulty. When the task required discriminating perfect symmetry from $90 \%$ symmetry (Experiment 2 difficult condition) - a task that previous work suggests may require serial scrutiny - accuracy was indeed very low. However, dividing the display into two parts and allowing the subjects to process them one by one did not improve performance. Thus, it seems clear that this task elicited relatively slow but still parallel processing with no evidence of capacity limitations (slow in the sense that very little processing can be accomplished in a given amount of time, thus yielding a small $d^{\prime}$ ). In Experiments 3 and 41 (density halved; area constant), overall performance was significantly better when the displays were presented successively, regardless of whether the task required detecting approximate symmetry (Experiment 3 ) or small asymmetries (Experiment 4) and regardless of difficulty. This result suggests that symmetry perception is always

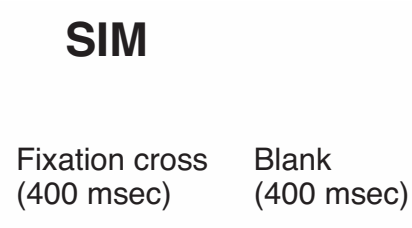

\section{SUCC}

Fixation cross Blank (400 msec) (400 msec)

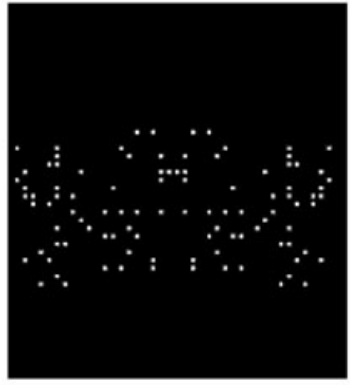

(100 msec)

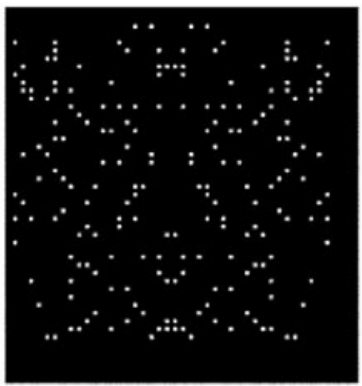

Mask (100 msec)

(100 msec)

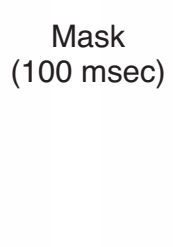

(300 msec)

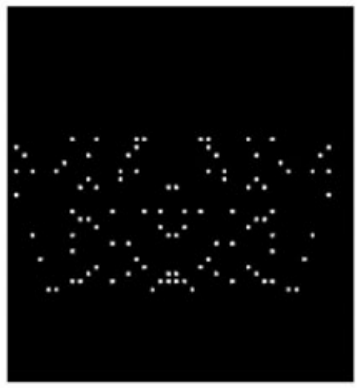

(100 msec)

Figure 2. The procedure in the SUCC and SIM conditions. In the SIM condition, the display was presented as a whole. In the SUCC condition, the display was divided into two parts (see text for details of division), with the second part presented $500 \mathrm{msec}$ after the first. 


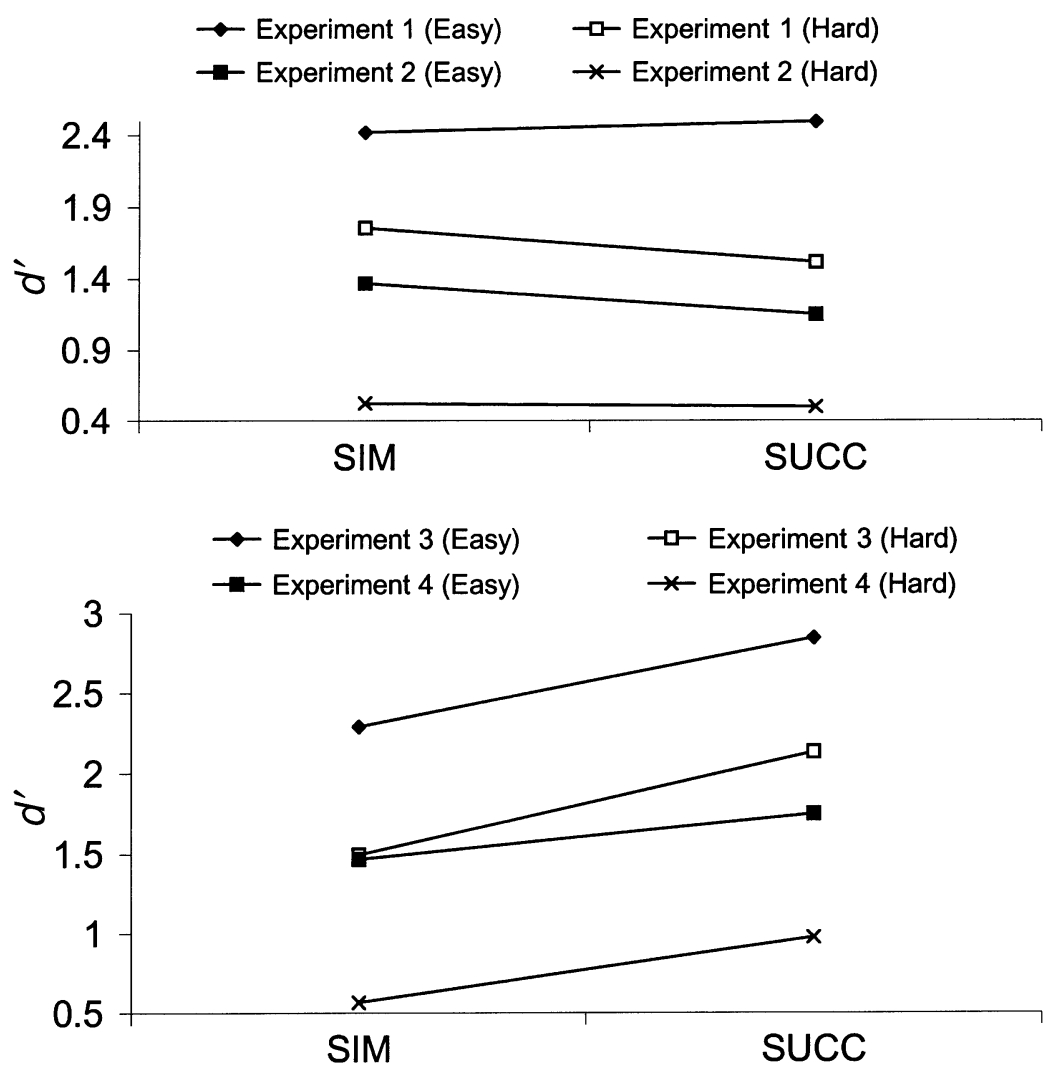

Figure 3. The data plotted as $d^{\prime}$. The SUCC condition is better than the SIM condition in Experiments 3 and 4, but not in Experiments 1 and 2.

coarse (in the sense that it cannot extract all the available information in a small area simultaneously) rather than focused within a small spatial extent.

A three-way ANOVA (factors: task type and difficulty; SUCC or SIM displays; density constant/area halved vs. density halved/area constant) was performed to analyze the data. Task type and difficulty were not analyzed as separate factors, because the meanings of "hard" and "easy" changed between detections of crude symmetry and small deviations from perfect symmetry, and therefore

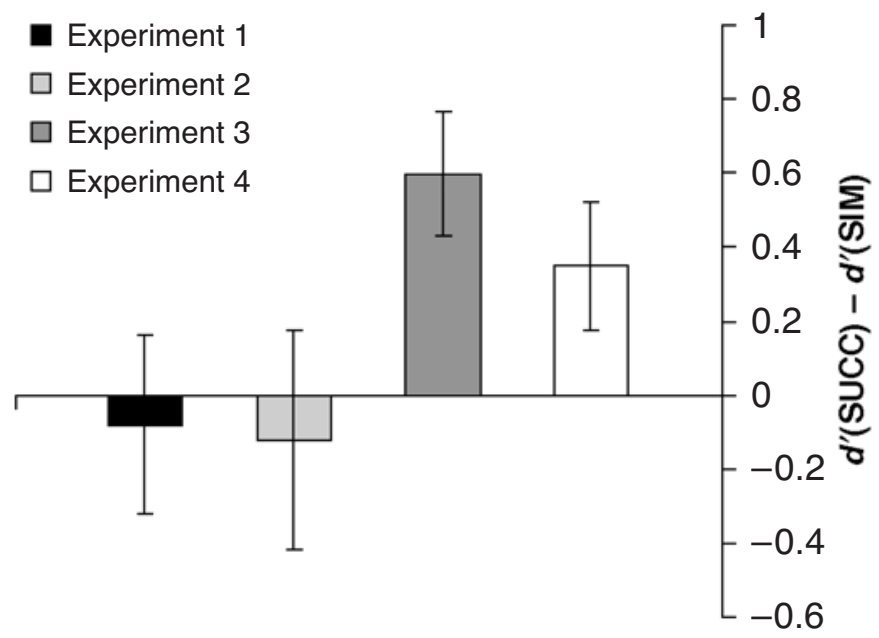

Figure 4. The advantage of SUCC over SIM, plotted as the difference of $d^{\prime}$ s and the confidence interval of this advantage. 
Table 1

One-Way ANOVA Comparison of $\boldsymbol{d}^{\prime}$ (SUCC) and $\boldsymbol{d}^{\prime}$ (SIM)

in Experiments 1-4

\begin{tabular}{ccccc}
\hline Experiment & Condition & $d^{\prime}(\mathrm{SUCC})-d^{\prime}(\mathrm{SIM})$ & $F$ value & $p$ \\
\hline 1 & Easy & 0.076 & $F(1,11)=0.124$ & n.s. \\
1 & Hard & -0.239 & $F(1,11)=4.735$ & n.s. \\
2 & Easy & -0.219 & $F(1,11)=1.701$ & n.s. \\
2 & Hard & -0.027 & $F(1,11)=0.051$ & n.s. \\
3 & Easy & 0.555 & $F(1,10)=41.740$ & $<.001$ \\
3 & Hard & 0.641 & $F(1,10)=38.995$ & $<.001$ \\
4 & Easy & 0.283 & $F(1,11)=5.111$ & $<.05$ \\
4 & Hard & 0.411 & $F(1,11)=20.537$ & $<.001$ \\
\hline
\end{tabular}

Note-The SUCC results are significantly better than those for SIM across all conditions in Experiments 3 and 4, but not in Experiments 1 and 2.

are incomparable. There was a significant interaction between the other two factors [SUCC or SIM displays; density constant/area halved vs. density halved/area constant; $\left.F(1,21)=40.90, M S_{\mathrm{e}}=0.10, p<.001\right]$, suggesting that the way of dividing stimuli does affect the existence of a SUCC/SIM advantage. There was no significant three-way interaction $\left[F(3,63)=1.00, M S_{\mathrm{e}}=0.11\right]$, suggesting that the interaction described above is not affected by the task type and difficulty. Both of these results confirm those in the paragraph above.

\section{DISCUSSION}

The experiments reported here indicate that the task type (detecting symmetry or asymmetry) and the precision required for accurate judgment do not affect whether capacity limitations appear in a symmetry detection task. Even when processing is comparatively slow and ineffective (given the display durations), it appears that symmetry is assessed in parallel across the display without a capacity limit. This result does not necessarily indicate that spatially serial "scrutiny" never plays a role in symmetry judgment experiments. Such scrutiny seems likely to occur whenever eye movements are permitted and when they are useful in overcoming the detrimental effects of eccentricity on spatial judgments. Within a given fixation, however, our data provide no evidence that symmetry judgments of any kind can be improved by focusing attention on a spatially defined subset of the display.

The globally parallel nature of symmetry perception confirms that it is likely a unique form of processing that differs from other mathematically similar forms of perceptual analysis (e.g., repetition detection; cf. van der Helm \& Leeuwenberg, 1996, for one possible mechanistic explanation of this difference).

Naturally, our conclusion that processing in different regions of the visual field operates in parallel does not imply that all regions of the visual field are equally influential in symmetry judgments. Indeed, there is some evidence (Jenkins, 1982, 1983) to suggest that a small area around the axis of symmetry may provide the strongest input (but see Tyler, 1999). There is no contradiction here: the notion of unlimited capacity parallel processing implies that there is no tradeoff between processing in one part of the visual field and processing in another, but it does not imply that all parts of the visual field have an equally important input into the overall judgment.

Our results also show a substantial benefit when different subsets of the dots in a given region can be processed during successive periods of time. The results show a density effect. To put it in another way, symmetry perception is coarse, in the sense that limited information can be extracted from a dense display.

This density effect potentially has several different causes. First, it may reflect the fact that some kind of attentional capacity is needed to extract information from any small area of the visual field. Second, it is also possible that, in dense displays, perception of spatial position may be impaired by lateral masking (sometimes termed visual crowding: Wolford \& Hollingsworth, 1974). However, it seems the lateral masking itself, previously believed to arise from limitations in early visual processing, may reflect attentional limitations (He, Cavanagh, \& Intriligator, 1996). A third possibility can be constructed based on ideas suggested by Tyler and Hardage (1996). These investigators proposed a theory that symmetry perception is largely mediated by computation of secondorder mechanisms which analyze the contrast envelope of the pattern as a whole. Because the contrast envelope varies only slightly in low-density displays but greatly in high-density displays, this theory might potentially explain the density effect reported above.

One possibility is that no attentional capacity is required for symmetry perception in small areas, and the density effect could occur for other reasons. Another is that there is always an attentional capacity limit for symmetry perception in a given small area. In either case, the kind of symmetry judgment required (detecting crude symmetry or a small deviation from perfect symmetry) and the task difficulty do not influence whether or not capacity limitations appear. Both pieces of evidence (global parallel processing and density effect) suggest there is no real difference in the underlying mechanisms used to perform crude symmetry detection and precise symmetry detection.

The two properties we infer from our results (no tradeoff between different regions of visual field and advan- 
tages when the amount of information packed into a small area is reduced) have been cited by two-stage process theorists as characterizing the first crude, preattentive stage of symmetry perception (S. E. Palmer \& Hemenway, 1978; Royer, 1981). Our results suggest that both of these properties hold even when the task is sufficiently difficult that one might expect it to force serial scrutiny (and thus reliance on the putative second stage of processing postulated by two-stage process theories). As noted above, with displays that remain present until observers respond, the occurrence of serial processing across different locations in the visual field is likely to reflect the role of eye movements rather than an inability to process different parts of a display in parallel with no capacity limit. Previous research has sometimes shown that certain kinds of strategic processing can take place when the observer has the opportunity to view the display for adequate periods of time, but not in the case of brief masked displays (Moore \& Egeth, 1998). Thus, although we cannot completely rule out the possibility of a scrutiny that occurs slowly, it at least seems clear that such a strategy is not a natural element of symmetry perception that can be used immediately.

\section{LIMITATIONS AND FUTURE DIRECTIONS}

Naturally, the experiments reported here were carried out in only one of several possible ways, and these methodological choices may have affected the outcome. For one thing, in Experiments 1 and 2, observers could not shift their attention in advance in SUCC displays, because these displays appeared in random locations and because SUCC and SIM displays were intermixed. If observers had been allowed to prepare fully for the location of each SUCC frame, their performance could possibly have been different. For another thing, the axis of symmetry was always vertical in our experiments, and hard and easy trials were separated into different blocks. It is conceivable that different results might occur in a task in which locating the axis of symmetry posed a challenge.

In summary, the experiments reported here indicate two interesting findings. First, symmetry appears to be assessed in parallel across the display without a capacity limit, although the question of whether the density effect reflects an attentional capacity is less clear. Second, and more importantly, the nature of symmetry perception does not change given different tasks, whether the task is easy or hard, detecting crude symmetry or a small deviation from perfect symmetry.

\section{REFERENCES}

BARLOW, H. B., \& ReEves, B. C. (1979). The versatility and absolute efficiency of detecting mirror symmetry in random dot displays. $\mathrm{Vi}$ sion Research, 19, 783-793.

DAKIN, S. C., \& HESS, R. F. (1997). The spatial mechanisms mediating symmetry perception. Vision Research, 37, 2915-2930.

DunCAN, J. (1980). The demonstration of capacity limitation. Cognitive Psychology, 12, 75-96.

Eckstein, M. P., Thomas, J. P., Palmer, J., \& Shimozaki, S. S. (2000).
A signal detection model predicts the effects of set size on visual search accuracy for feature, conjunction, triple conjunction, and disjunction displays. Perception \& Psychophysics, 62, 425-451.

ERIKSEN, C. W., \& SPENCER, T. (1969). Rate of information processing in visual perception: Some results and methodological considerations. Journal of Experimental Psychology, 79(2, Pt. 2), 1-16.

Foster, D. H. (1991). Operating on spatial operations. In R. J. Watt (Ed.), Pattern recognition by man and machine (pp. 50-58). Basingstoke, U.K.: Macmillan.

GARDNer, G. T., \& JosePh, D. J. (1975). Parallel perceptual channels at "deep" processing levels. Bulletin of the Psychonomic Society, 6, 658-660.

Gurnsey, R., Herbert, A. M., \& Kenemy, J. (1998). Bilateral symmetry embedded in noise is detected accurately only at fixation. $\mathrm{Vi}$ sion Research, 38, 3795-3803.

He, S., Cavanagh, P., \& Intriligator, J. (1996). Attentional resolution and the locus of visual awareness. Nature, 383, 334-337.

Hochstein, S., \& Ahissar, M. (2002). View from the top: Hierarchies and reverse hierarchies in the visual system. Neuron, 36, 791-804.

JENKINS, B. (1982). Redundancy in the perception of bilateral symmetry in dot textures. Perception \& Psychophysics, 32, 171-177.

JENKIns, B. (1983). Spatial limits to the detection of transpositional symmetry in dynamic dot textures. Journal of Experimental Psychology: Human Perception \& Performance, 9, 258-269.

JuLESZ, B. (1981). Figure and ground perception in briefly presented isodipole textures. In M. Kubovy \& J. Pomerantz (Eds.), Perceptual organization (pp. 27-54). Hillsdale, NJ: Erlbaum.

Locher, P. J., \& Wagemans, J. (1993). Effects of element type and spatial grouping on symmetry detection. Perception, 22, 565-587.

Moore, C. M., \& Egeth, H. (1998). How does feature-based attention affect visual processing? Journal of Experimental Psychology: Human Perception \& Performance, 24, 1296-1310.

OLIVERS, C. N. L., \& VAN DER HELM, P. A. (1998). Symmetry and selective attention: A dissociation between effortless perception and serial search. Perception \& Psychophysics, 60, 1101-1116.

PALMER, J. (1994). Set-size effect in visual search: The effect of attention is independent of the stimulus for simpler tasks. Vision Research, 34, 1703-1721.

PALMER, S. E., \& Hemenway, K. (1978). Orientation and symmetry: Effects of multiple, rotational, and near symmetries. Journal of Experimental Psychology: Human Perception \& Performance, 4, 691702.

PAshler, H. (1990). Coordinate frame for symmetry detection and object recognition. Journal of Experimental Psychology: Human Perception \& Performance, 16, 150-163.

PASHLER, H. (1998). The psychology of attention. Cambridge, MA: MIT Press.

Rainville, S. J. M., \& Kingdom, F. A. A. (2000). The functional role of oriented spatial filters in the perception of mirror symmetryPsychophysics and modeling. Vision Research, 40, 2621-2644.

RoYer, F. L. (1981). Detection of symmetry. Journal of Experimental Psychology: Human Perception \& Performance, 7, 1186-1210.

SHAW, M. L. (1984). Division of attention among spatial locations: A fundamental difference between detection of letters and detection of luminance increments. In H. Bouma \& D. G. Bouwhuis (Eds.), Attention and performance $X$ (pp. 109-121). Hillsdale, NJ: Erlbaum.

SHIFFrIN, R. M., \& GARDNER, G. T. (1972). Visual processing capacity and attentional control. Journal of Experimental Psychology, 93, 7882.

TAPiOVAaRA, M. (1990). Ideal observer and absolute efficiency of detecting mirror symmetry in random images. Journal of the Optical Society of America A, 7, 2245-2253.

Treisman, A., \& Gelade, G. (1980). A feature integration theory of attention. Cognitive Psychology, 12, 97-136.

Tyler, C. W. (1999). Human symmetry detection exhibits reverse eccentricity scaling. Visual Neuroscience, 16, 919-922.

Tyler, C. W., \& HarDAGE, L. (1996). Mirror symmetry detection: Predominance of second-order pattern processing throughout the visual field. In C. W. Tyler (Ed.), Human symmetry perception and its computational analysis (pp. 157-172). Utrecht: VSP.

VAN Der Helm, P. A., \& Leeuwenberg, E. L. J. (1996). Goodness of 
visual regularities: A nontransformational approach. Psychological Review, 103, 429-456.

WaGemans, J. (1995). Detection of visual symmetries. Spatial Vision, 9, 9-32.

WENDEROTH, P. (1994). The salience of visual symmetry. Perception, 23, 221-236.

WENDEROTH, P. (1997). The effects on bilateral symmetry detection of multiple symmetry, near symmetry, and axis orientation. Perception, 26, 891-904.

WOLFORD, G., \& HOLLINGSWORTH, S. (1974). Lateral masking in visual information processing. Perception \& Psychophysics, 16, 315-320.

\section{NOTE}

1. In Experiment 3, the data of 1 subject were excluded from analysis of $d^{\prime}$, because in one condition her accuracy for symmetric display was 1 , and thus her $d^{\prime}$ would have been infinity. This result occurred in the SUCC condition, so it does not undermine our conclusion.

(Manuscript received December 5, 2002; revision accepted for publication October 20, 2003.) 